intrauterine fibroids, myomas or the foetal limbs could result in deformation.

In the case described the history of oligohydramnios may have contributed to skull depression by bringing the foetal skull into contact with the solid structures within the maternal pelvis.

Proposed treatments for congenital SD include conservative management, surgical and non-surgical interventions. The majority of skull depressions resolve spontaneously, therefore as in this case, in the absence of neurological symptoms a conservative approach of a six-month observation period is recommended.

\section{P483 HOME OXYGEN REFERRAL IN PREMATURE NEONATES BORN IN UMHL OVER TWO YEARS PERIOD}

Aisha ljaz*, Mohammed Abu Bakar, Rizwan Khan, Ghulam Raza. University Maternity Hospital, Limerick, Ireland

\subsection{6/archdischild-2019-epa.819}

Background Bronchopulmonary dysplasia(BPD) has been a challenging condition for neonatologists(1). Prematurely born infants who had BPD may require supplementary oxygen at home for many months(2).

Objectives To determine the number of premature babies discharged on home oxygen over two years period in UMHL and to confirm about average gestation age at which babies were self ventilating in air.

Methodology Retrospective data of 38 babies equal to or less than 29 weeks of gestation was collected, born in UMHL from February 2016 to February 2018. Different variables were studied including Gestation at birth, Birth weight, Date of admission, Date of discharge, Gestation for self ventilation in air, Gestation of home oxygen referral and gestation at discharge. Management of Chronic Lung Disease in the form of steroids, diuretics, patent ductus arteriosus(PDA) treatment and number of blood transfusions were also looked upon.

Results Total 38 babies born in UMHL from February 2016 to February 2018, equal to or less than 29 weeks of gestation. $26 \%(n=10)$ babies were excluded from the study including $21 \%(n=8)$ babies died, $3 \%(n=1)$ chart missing and $3 \%(n=1)$ baby lost follow up. $71 \%(\mathrm{n}=20)$ were born in the gestation range of 26 to 28 weeks and $70 \%(n=19)$ were between birth weight of 750 grams to 1250 grams. $59 \%(n=16)$ babies were self ventilating in air between 31 to 35 weeks of gestation compared to $11 \%(n=3)$ who took over 41 weeks of corrected gestational age. $4 \%(n=1)$ babies were not able to wean off from oxygen over two years period. $29 \%(n=8)$ of the babies didn't need any intervention (Diuretics, Steroids, PDA management, Blood transfusion) during their stay in NICU while $29 \%(n=8)$ needed just one intervention. $43 \%(n=12)$ of the babies needed two or more interventions to achieve self ventilation in air.

Conclusion Only one baby out of total 28 was discharged on home oxygen over two years period and most of the babies were able to achieve self ventilation in air between 31 st to 35 th weeks of gestation.

\section{REFERENCES}

1. Colby L. Day, Rita M. Ryan. Bronchopulmonary dysplasia: new becomes old again! Pediatric Research2017;81:210-213
2. Greenough A, Long-term respiratory consequences of premature birth at less than 32 weeks of gestation, Early Human Development 2013 Oct;89 Suppl 2: S25-7

\section{P484 THE USE OF MASSAGE THERAPY FOR TREATMENT OF NEONATAL HYPERBILIRUBINEMIA : A SYSTEMATIC REVIEW AND NETWORK META-ANALYSIS}

${ }^{1}$ Mohammed Abdelrahman*, ${ }^{2}$ Nguyen Lam Vuong, ${ }^{3}$ Gehad Tawfik, Do Phuc Nhu Nguyen ${ }^{4} 2,{ }^{5}$ Le Van Thanh, Muhammed Khaled ${ }^{6},{ }^{7}$ Marwa Mansour, ${ }^{8}$ Pham Diep Thuy Duong, ${ }^{9}$ Yifan Liang, ${ }^{9}$ Kaipeng Liu, ${ }^{10}$ Kenji Hirayama, ${ }^{11}$ Nguyen Tien Huy. ${ }^{1}$ NICU, kuwai, Kuwait; ${ }^{2} 3$ University of Medicine and Pharmacy at Ho Chi Minh City, Ho Chi Minh City, Vietnam; ${ }^{3}$ Faculty of Medicine, Ain Shams University, Cairo, Egypt; ${ }^{4}$ Epidemiology Department, The Institute of Public Health, Ho Chi Minh City, Vietnam; ${ }^{5}$ Faculty of Medicine, University of Medicine and Pharmacy at Ho Chi Minh City, Ho Chi Minh City, Vietnam; ${ }^{6}$ Faculty of Medicine, University of Tripoli, Tripoli, Libya; ${ }^{7}$ Ministry of Health and Population, Cairo, Egypt; ${ }^{8}$ University of Medicine and Pharmacy at Ho Chi Minh City, Ho Chi Minh City, Vietnam; ${ }^{9}$ China Medical University, China, China; ${ }^{10}$ Department of Immunogenetics, Institute of Tropical Medicine (NEKKEN), Leading Graduate School Program, and Graduate School of Biomedical Sciences, Nagasaki University, Nagasaki, Japan; "115Department of Clinical Product Development, Institute of Tropical Medicine (NEKKEN), Leading Graduate School Program, and Graduate School of Biomedical Sciences, Nagasaki University, Nagasaki, Japan

\subsection{6/archdischild-2019-epa.820}

Background Neonatal jaundice is a common health problem which is expected to be seen in $60-80 \%$ of healthy newborns, with $10 \%$ occurrence of hyperbilirubinemia in neonates during first week of life. Massage therapy has been practiced for centuries to gain health benefits in infants. We aimed to evaluate effectiveness of various types of infant massage in reduction of neonatal jaundice and the need for phototherapy (PT).

Methods On 26 March 2016, we conducted electronic search term in nine databases, with inclusion of randomized controlled trials (RCTs) that investigated newborn babies receiving massage therapy with documented total bilirubin before and after the treatment. Study protocol was registered on PROSPERO, CRD42016049025. Quality assessment was performed using Cochrane risk of bias tool. Frequentist network metaanalysis (NMA) and meta-analysis (MA) were used to compare all outcomes by each day of follow-up.

Results With 363 studies initially identified, 32 were eligible for qualitative analysis and 29 for quantitative analysis. For neonates requiring PT, our NMA results showed that in 3rd day of life, massage and phototherapy (MP) was significantly the most effective in reduction of serum bilirubin level, while acupressure massage and phototherapy (AMP) was the least compared to by phototherapy only (P), with MD, 95\%CI ($1.16[-1.61 ;-0.72], 1.28[0.79 ; 1.77]$ respectively). In $14^{\text {th }}$ day of life, bilirubin level decreased the most in massage with enema and phototherapy (MEP), while MP has no significant difference compared to P. Our MA results in $4^{\text {th }}$ day of life, indicated that MP had a significant decrease in bilirubin level compared with P, with MD 95\%CI (-2.23 [-3.43; -1.03]). In summary comparison with $\mathrm{P}, \mathrm{MP}$ was the most in decreasing bilirubin level in most of days, with the biggest decrease being in $4^{\text {th }}$ day, while MEP was significantly the most in $14^{\text {th }}$ day. For neonates not requiring PT, our NMA showed a significant decrease in bilirubin level with massage and bathing, acupressure massage, and massage 3-4 times/days (M2) groups in 3rd day of life, and with massage 1-2 times/days (M1) and M2 groups in 4th day. MEP group had a significantly longer duration of PT compared with P, with MD 95\%CI (29.09 [9.76; 